\title{
Towards the Distributed Burning Regime in Turbulent Premixed Flames
}

\author{
A. J. Aspden ${ }^{1,2}$, M. S. Day² and J. B. Bell ${ }^{2}$ \\ ${ }^{1}$ School of Engineering, Newcastle University, Stephenson Building, \\ Claremont Road, Newcastle-Upon-Tyne, NE1 7RU, UK \\ ${ }^{2}$ Center for Computational Sciences and Engineering, Lawrence Berkeley National Laboratory, \\ MS50A-3100, 1 Cyclotron Road, Berkeley, CA 94720, USA
}

25th June 2018

\begin{abstract}
Three-dimensional numerical simulations of canonical statistically-steady statistically-planar turbulent flames have been used in an attempt to produce distributed burning in lean methane and hydrogen flames. Dilatation across the flame means that extremely large Karlovitz numbers are required; even at the extreme levels of turbulence studied (up to a Karlovitz number of 8767) distributed burning was only achieved in the hydrogen case. In this case, turbulence was found to broaden the reaction zone visually by around an order of magnitude, and thermodiffusive effects (typically present for lean hydrogen flames) were not observed. In the preheat zone, the species compositions differ considerably from those of one-dimensional flames based a number of different transport models (mixture-averaged, unity Lewis number, and a turbulent eddy viscosity model). The behaviour is a characteristic of turbulence dominating non-unity Lewis number species transport, and the distinct limit is again attributed to dilatation and its effect on the turbulence. Peak local reaction rates are found to be lower in the distributed case than in the lower Karlovitz cases but higher than in the laminar flame, which is attributed to effects that arise from the modified fuel-temperature distribution that results from turbulent mixing dominating low Lewis number thermodiffusive effects. Finally, approaches to achieve distributed burning at realisable conditions are discussed; factors that increase the likelihood of realising distributed burning are higher pressure, lower equivalence ratio, higher Lewis number, and lower reactant temperature.
\end{abstract}

\section{Introduction}

The distributed burning regime of turbulent premixed flames represents the limiting case where flame propagation is driven by turbulent mixing rather than molecular diffusion [Aspden et al., 2011a], and corresponds to the small-scale turbulence limit [Peters, 2000, Damköhler, 1940]; the Reader is referred to Aspden et al. [2011a] for a more detailed review of the use of the term distributed burning. The feature that distinguishes burning in the distributed mode is that turbulent eddies comparable with the reaction zone thickness can mix faster than the flame can burn. Experimentally, there is some evidence of what will be referred to here as "transitionally-distributed" burning, particularly in high-speed piloted lean-to-stoichiometric methane jet flames; see, for example, Dunn et al. [2010], Zhou et al. [2017], Skiba et al. [2018], and the references therein. Previous numerical studies of transitionally-distributed flames include Poludnenko and Oran [2010], Aspden et al. [2011a], Savard and Blanquart [2015], Lapointe et al. [2015], Nilsson et al. [2018], Wang et al. [2018], and the references therein. All of these studies have demonstrated flames with broadened preheat zones, but none have shown broadening of the actual reaction zone by turbulence alone. Skiba et al. [2018] referred to this mode of burning as "broadened preheat thin reactions".

Distributed burning has been observed numerically in an astrophysical context [Aspden et al., 2008a], where a single-step reaction model was used to represent thermonuclear fusion in a type Ia supernova flame. Subsequently, Aspden et al. [2010] demonstrated scaling laws for distributed 
supernova flames following Damköhler [1940] and Peters [2000], along with the so-called " $\lambda$-flame" regime; the combination of large Karlovitz and Damköhler numbers gives rise to flames simultaneously in the small-scale and large-scale limits. The resulting regime has such a large range of turbulent scales that the flame burns in the distributed mode, but the larger scales are unable to mix before the flame burns; this mode of burning aligns with that predicted by Zimont [1979] (which we argue requires the distributed transition to broaden the flame), and the local turbulent flame thickness $\lambda$ corresponds to the Zimont length [Peters, 2000] at that Karlovitz number. These supernova studies used an idealised configuration capable of subjecting the flame to arbitrary levels of turbulence favourable for distributed burning (the Reynolds number in a supernova can be in excess of $10^{10}$ and the Mach number around $10^{-5}$ ); realisable conditions for distributed burning in terrestrial chemical flames are yet to be established.

In the present paper, lean premixed methane and hydrogen flames have been simulated with extreme levels of turbulence (rms velocity fluctuations exceeding four hundred times the laminar flame speed). At the highest turbulence levels, the hydrogen flame has been found to present substantial broadening of the reaction zone, but even with such intense turbulence, the methane flame did not. Despite the abstracted configuration and unrealistic conditions, this hydrogen flame represents the transition to distributed burning expected of a terrestrial chemical flame if suitable conditions can be contrived (i.e. at the same Karlovitz and Damköhler numbers). Phenomenological observations are first presented, followed by consideration of global consumption speeds, flame thickening, and conditional means of heat release and species mass fractions. The paper concludes with a discussion of the distributed burning regime and potential conditions required to realise distributed burning at realisable conditions.

\subsection{A note on Karlovitz number}

The classical Richardson/Kolmogorov picture of turbulence is a cascade of turbulent eddies from the energy-containing large scales through the inertial subrange to the dissipation subrange. If it is assumed that turbulence is sufficient for the inertial subrange to extend down to the flame scale, (equivalently, the flame is in the thin reaction zone, or the Karlovitz number is larger than unity), then as in the classical picture, there are energy-containing large scales responsible for the supply of kinetic energy through the inertial subrange to the flame scale, where (unlike the non-reacting case) dilatation modifies the turbulent structure close to the flame before it reaches a classical dissipation subrange. Energy is still dissipated by viscosity at small scales, but not at the usual Kolmogorov length scale that would be expected in a constant density flow; see Towery et al. [2016] for a more detailed consideration of energy spectra in premixed flames.

For this reason, it seems illogical to define the Karlovitz number (in the sense of being characteristic of turbulence-flame interaction at the flame scale) in terms of Kolmogorov scales; these scales simply aren't representative of the physical processes taking place. Following the Kolmogorov similarity hypotheses, the universal equilibrium range is determined by the energy dissipation rate $(\varepsilon)$ and viscosity $(\nu)$. The second similarity hypothesis gives rise to the inertial subrange independent from $\nu$, determined solely by $\varepsilon$. This second hypothesis appears to be relevant to a turbulent premixed flame, which suggests a relevant dimensionless parameter can be defined as

$$
\Pi_{\varepsilon}^{2}=\frac{\varepsilon}{\varepsilon_{F}}=\frac{u^{\prime 3}}{l} \frac{l_{F}}{s_{F}^{3}}
$$

where $u^{\prime}$ and $l$ are the rms velocity fluctuation and integral length scale respectively, and $s_{F}$ and $l_{F}$ are the characteristic speed and thermal thickness of the flame (and $\varepsilon_{F}=s_{F}^{3} / l_{F}$ ). This quantity is the same as a more conventionally-defined Karlovitz number based on the Kolmogorov time scale and by assuming a unity flame Reynolds number i.e. $s_{F} l_{F}=\nu$ [e.g. Peters, 2000], but the argument here is that (1) is the appropriate dimensionless parameter that characterises the strength of turbulence at the flame scale in the thin reaction zone and above, (specifically, $u_{F} / s_{F}=\Pi_{\varepsilon}^{2 / 3}$, where $u_{F}$ is the velocity associated with a turbulent eddy in the inertial subrange at the flame scale), and so should be considered the starting point, rather than resulting from any assumption. It is not only important to 
distinguish this quantity from the conventional definition involving Kolmogorov scales, but also from the definition used in the flamelet regimes as a measure of flame stretch; these definitions represent fundamentally different physical interactions. Despite the distinction, $\Pi_{\varepsilon}$ will still be referred to here as a Karlovitz number (with $\mathrm{Ka} \equiv \Pi_{\varepsilon}$ ) and the implicit understanding that it is not a measure of stretch, nor does it involve viscosity.

\section{Simulation Details}

\subsection{Numerical solver}

The numerical solver used here is based on the well-established low Mach number formulation of the reacting flow equations [Day and Bell, 2000, Nonaka et al., 2012]. The fluid is treated as a mixture of perfect gases, and a mixture-averaged model is assumed for diffusive transport. A source term is used in the momentum equation to establish and maintain turbulence with the desired properties [Aspden et al., 2008b]. The chemical kinetics and transport are modelled using the hydrogen mechanism of $\mathrm{Li}$ et al. [2004] consisting of 9 species with 21 fundamental reactions, and the GRIMech 3.0 methane mechanism [Frenklach et al., 1995] with the nitrogen reactions removed, resulting in 35 species and 217 reactions. These evolution equations are supplemented by CHEMKIN-compatible databases for thermodynamic quantities, and transport properties computed using EGLIB [Ern and Giovangigli, 1996].

This solver is capable of running implicit large eddy simulation (ILES); non-oscillatory finitevolume schemes such as this are able to dissipate kinetic energy numerically at the grid scale in a stable and physical manner without resolving all the way down to the Kolmogorov length scale (see Grinstein et al. [2007] for a review). In the present simulations, especially at the highest turbulence intensities, there is some reliance on this ILES capability in that the Kolmgorov length scale in the reactants is not resolved on the grid. The performance of this solver in such under-resolved conditions was characterised in Aspden et al. [2008b], and further details are given below.

\subsection{Simulation configuration}

Following our previous studies [e.g. Aspden et al., 2011a, 2015, 2016], a canonical periodic-box configuration was used, where a lean premixed flame was allowed to propagate through maintained zero-mean homogeneous isotropic turbulence. All simulations were run at atmospheric conditions in a high aspect ratio domain, with periodic lateral boundary conditions, a free-slip base and outflow at the top. Lean premixed hydrogen (equivalence ratio $\varphi=0.4$, Lewis number Le $\approx 0.37$ ) and methane $(\varphi=0.7$, Le $\approx 1.0)$ were considered. The freely-propagating hydrogen flame speed and thickness are $s_{F}=0.474 \mathrm{~m} / \mathrm{s}$ and $l_{F}=410 \mu \mathrm{m}$; the laminar methane flame speed and thickness are $s_{F}=0.189 \mathrm{~m} / \mathrm{s}$ and $l_{F}=660 \mu \mathrm{m}$. It was shown in Aspden et al. [2008b] that the forcing approach gives approximately 10 integral length scales across the domain width. In all cases the length scale ratio is $\Lambda=l / l_{F}=1$, consistent with our previous studies (e.g. Aspden et al. [2017]). Three further Karlovitz numbers have been considered $\mathrm{Ka}=108,974$, and 8767 , which correspond to velocity ratios $\Upsilon=u^{\prime} / s_{F}=22.7$, 98.3, and 425, respectively. Present simulations and previous simulations are shown respectively as plusses and circles on a regime diagram in figure 1, where the critical Karlovitz number for transition to distributed burning has been shown in grey, and placed higher than is usual; it should be noted that this critical Karlovitz number is strongly dependent on the reactant conditions.

Most simulations were conducted with a domain size of $10 l_{F} \times 10 l_{F} \times 80 l_{F}$ discretised on a grid of $192 \times 192 \times 1536$ computational cells; the hydrogen case with the highest Ka required a larger domain $\left(10 l_{F} \times 10 l_{F} \times 120 l_{F} ; 192 \times 192 \times 2304\right.$ cells $)$ to accommodate the growth of the flame brush. This resolution corresponds to 19.2 cells across a thermal thickness, which is more than sufficient to resolve these chemical mechanisms [Aspden et al., 2011a, 2016]. At such high turbulence levels, the Kolmogorov length scale is between 44 and 544 times smaller than the thermal thickness of the flame; it is not resolved on this grid and so these simulations cannot be considered DNS in the sense that the term implies well-resolved down to the Kolmogorov length scale. The effective Kolmogorov length scale (see Aspden et al. [2008b] for details) can be evaluated for this solver, and was found to range 


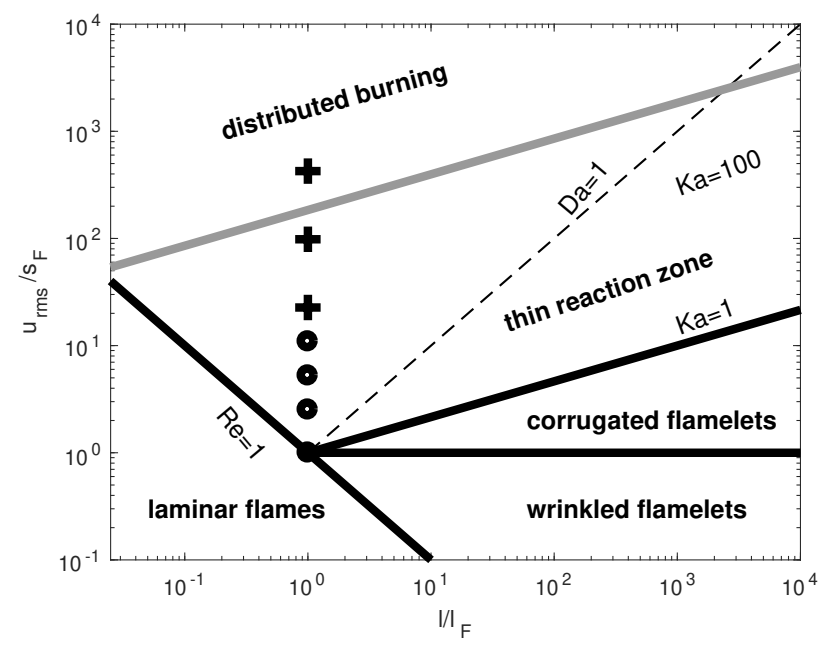

Figure 1: Turbulent premixed regime diagram showing the simulations in the present study (plusses) along with our previous simulations (circles) at lower Ka [Aspden et al., 2017]; note that the critical Karlovitz number is shown in grey, and has been placed higher than is usual.

between 38 and 67 times smaller than the thermal thickness, which indicates that the turbulence that interacts with the flame (i.e. at the flame scale) is sufficiently well-resolved (and is maintained by the momentum source term through the inertial cascade). Moreover, as previously argued, turbulence is strongly affected by dilatation, therefore, close to the flame, the Kolmogorov length is not the value that would be expected from the classical cascade; we argue that scales not represented on the grid have an inconsequential effect on burning regime and leading-order flame response to turbulence, and resolving them would be a waste of computational effort. The simulation parameters are given in table 1, where $\mathrm{Ka}_{\eta}=t_{F} / t_{\eta}$ denotes the more conventional Karlovitz number as the ratio of flame time $\left(t_{F}=l_{F} / s_{F}\right)$ to Kolmgorov times $\left(t_{\eta}=\eta / u_{\eta}\right), \operatorname{Re}=u^{\prime} l / \nu$ is the Reynolds number, and the suffix $e$ denotes effective quantities (i.e. using $\eta_{e}$ and $\nu_{e}$ ) evaluated following Aspden et al. [2008b]; note that all values are based on reactant conditions.

To establish the consequences of this lack of resolution on the turbulence, simulations of nonreacting homogeneous isotropic turbulence have been run at four times the resolution extending the length scales at each end of the spectrum separately. These simulations are presented in Appendix 4.2 and demonstrate that the use of maintained turbulence ensures the turbulence at the flame scale is the same as that that would have originated from a larger integral length scale at the same Karlovitz number (i.e. has the correct energy dissipation rate) despite the limited inertial subrange, and that the scales that are deficient are sufficiently smaller than the flame scale that we argue can be considered inconsequential. The effective Reynolds number is naturally lower, but the Karlovitz number (appropriately defined as above) is unaffected because the energy dissipation rate is unaffected by the lack of small-scale resolution. Note that it is this specific numerical approach (i.e. ILES-capable) that means the apparent lack of resolution does not invalidate the results; approximately twenty computational cells across the flame thermal thickness is sufficient.

\begin{tabular}{c|ccc|ccc} 
Case & $\mathrm{CH}_{4}(108)$ & $\mathrm{CH}_{4}(974)$ & $\mathrm{CH}_{4}(8767)$ & $\mathrm{H}_{2}(108)$ & $\mathrm{H}_{2}(974)$ & $\mathrm{H}_{2}(8767)$ \\
\hline$\Upsilon$ & 22.7 & 98.3 & 425 & 22.7 & 98.3 & 425 \\
$\mathrm{Ka}$ & 108 & 974 & 8,767 & 108 & 974 & 8,767 \\
$\mathrm{Da}$ & 0.0440 & 0.0102 & 0.00235 & 0.0440 & 0.0102 & 0.00235 \\
$\mathrm{Ka} \eta$ & 285 & 2,561 & 23,047 & 350 & 3,149 & 28,339 \\
$\mathrm{Ka}_{\eta_{e}}$ & 257 & 1,486 & 6,955 & 289 & 1,538 & 7,012 \\
$\mathrm{Re}$ & 157 & 679 & 2,938 & 237 & 1,027 & 4,442 \\
$\mathrm{Re}_{e}$ & 128 & 229 & 268 & 162 & 245 & 272
\end{tabular}

Table 1: Simulation parameters (based on reactant conditions). 
An additional simulation was also run to establish the consequences of under-resolving the Kolmogorov length scale in the methane case at $\mathrm{Ka}=8767$, which was achieved by adding a level of adaptive mesh refinement (AMR) and restarting a calculation from a steady-state check point (thereby having nearly 40 computational cells across the laminar thermal thickness); the results are presented in Appendix B. The turbulent flame speed was found to be almost unchanged with increased resolution, conditional means of fuel consumption rate and heat release were indistinguishable between resolutions, but there were subtle differences between the thickening metric (based on temperature gradients). The higher resolution simulation lends further support to our argument that the turbulent scales that are not resolved on the grid are inconsequential as far as the flame physics is concerned.

It should be further stressed that these simulations are numerical experiments, and this set of values is not realisable experimentally; in particular, the low Mach number approximation is exploited here to preclude strong compressibility effects and prevent any potential detonation [e.g. Poludnenko and Oran, 2010]. Note in particular that the low Mach number approximation is not valid at these conditions; these simulations are not intended as a true representation of how these flames would respond, but how turbulent flames at the same Karlovitz and Damköhler numbers would respond if conditions could be constructed at low Mach number. Furthermore, viscous heating has not been included, which would have the potential to lead to a significant rise in reactant temperature as a result of continual energy injection by the momentum forcing term. Despite the limitations of this configuration, especially at the highest $\mathrm{Ka}$, these simulations capture turbulence-flame interactions during the transition towards the limiting case of flame propagation driven by turbulent mixing, i.e. distributed burning, and are of significant interest and relevance to the transition away from the thin reaction zone with increasing levels of turbulence. Possible steps to realise distributed burning experimentally are discussed in section 4.

\section{Results}

\subsection{Flame response overview}

A general overview of flame response to high Ka turbulence is presented in figure 2, which depicts slices of fuel mass fraction, temperature, fuel consumption rate (FCR), and heat release rate (HR), normalised by the corresponding laminar values (note for for hydrogen, the FCR and HR are normalised by ten times the laminar value to allow for the enhanced reaction rates due to the thermodiffusive instability).

Methane at $\mathrm{Ka}=108$ appears to be similar to moderate $\mathrm{Ka}$ [see the $\mathrm{Ka}=36$ case in Aspden et al., 2016]; the flame surface is convoluted but smooth, generally similar to the laminar flame, with a decrease in reaction rates correlated with high positive curvature, which was attributed to atomic hydrogen diffusion [Echekki and Chen, 1996, Aspden et al., 2016]. The temperature field shows clear evidence of turbulent mixing, but restricted to the preheat region.

Methane at $\mathrm{Ka}=974$ continues the trend of increased turbulent mixing in the preheat region, but the reaction rates appear to be changing; while not immediately apparent in the image, the peak value exceeds the laminar flame values in places, and there appears to be greater variability along the flame surface, which is becoming more convoluted - again, this can be attributed to atomic hydrogen diffusion [Echekki and Chen, 1996, Aspden et al., 2016].

Methane at $\mathrm{Ka}=8767$ shows different behaviour than the lower Ka cases; in addition to the mixing observed in the preheat region, there are indications in the temperature field that there is an onset of turbulent mixing in the post-flame region. Interestingly, the reaction rates appear slightly increased in places (see magenta/white regions; an enlarged image is shown in figure 3), but are not broadened by turbulence.

Hydrogen at $\mathrm{Ka}=108$ presents significant thermodiffusively-unstable behaviour [e.g. Trouvé and Poinsot, 1994, Baum et al., 1994], and continues the trend from that observed in Aspden et al. [2011a, 2015]; turbulence exaggerates the thermodiffusive instability, creating small-scale structures with higher curvature than at lower Ka, resulting in more intense burning over a broad flame brush (localised heat release rates in excess of fifteen times the laminar value are observed; shown by the white regions). The decorrelation between fuel consumption and heat release rates (reported by Chen 


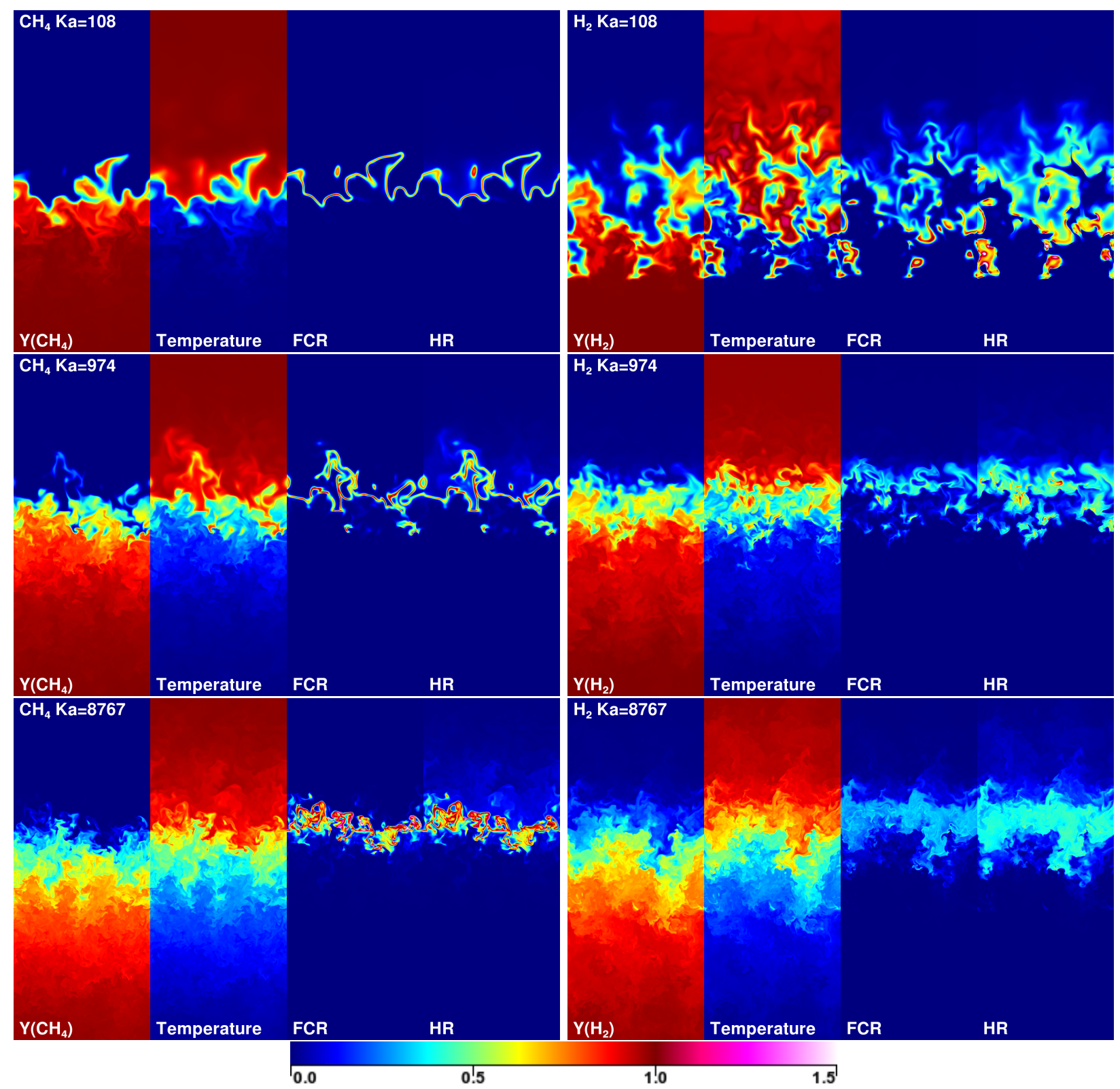

Figure 2: Slices of fuel mass fraction, temperature, fuel consumption rate, and heat release for $\mathrm{CH}_{4}$ and $\mathrm{H}_{2}$ flames at $\mathrm{Ka}=108,974$ and 8767, respectively. Each panel of each image shows $20 l_{F} \times 50 l_{F}$ (note that periodicity has been exploited to stitch together $x-z$ and $y-z$ planes to show more flame surface).

and Im [2000] and Aspden et al. [2015], and demonstrated to be due to atomic hydrogen diffusion Aspden [2017]) is present, further indicating persistence of preferential diffusion at this Ka. Superadiabatic temperatures still exist in the near post flame region. The temperature field presents limited turbulent mixing; the thermodiffusive instability leads to a resistance to turbulent mixing.

At $\mathrm{Ka}=974$, the hydrogen flame presents the first evidence of a change in behaviour; the thermodiffusively unstable structures at lower $\mathrm{Ka}$ are no longer observed, reaction rates have generally decreased, and the temperature field shows significant evidence of turbulent mixing in the preheat region.

At the highest Ka, the hydrogen flame presents significantly different behaviour to all of the other cases; there is now substantial turbulent mixing throughout the flame, there is no evidence of the thermodiffusive instability, the reaction rates are substantially lower than the other two hydrogen cases, and distributed relatively smoothly across a region that is over ten thermal thicknesses across. This behaviour is consistent with the distributed supernova flame presented in Aspden et al. [2008a]; 


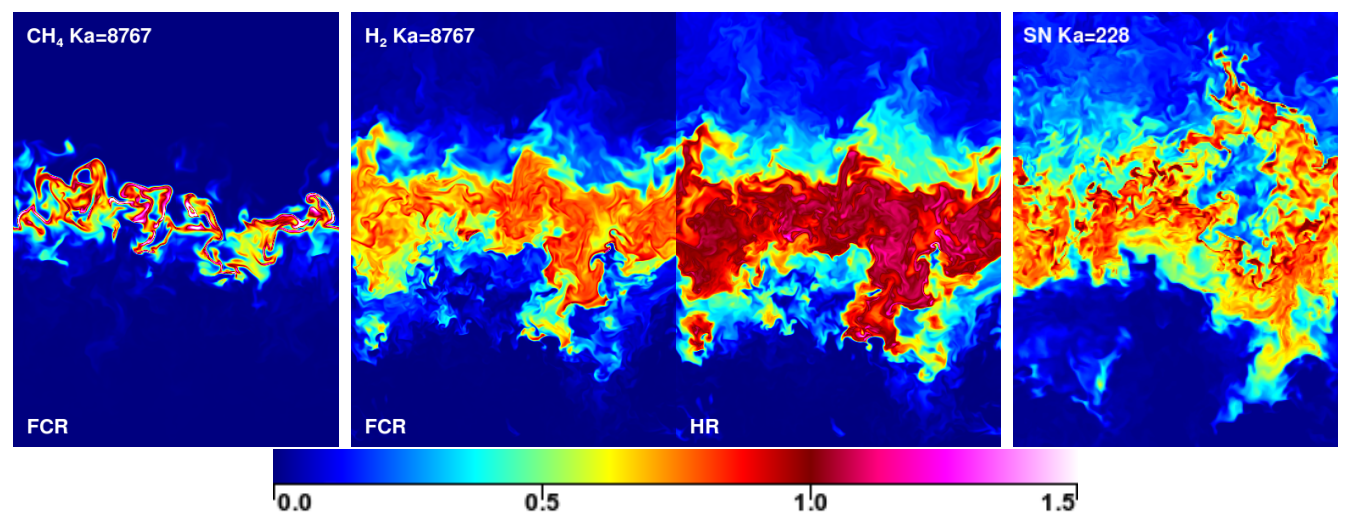

Figure 3: Slices of FCR for $\mathrm{CH}_{4}$, and FCR and $\mathrm{HR}$ for $\mathrm{H}_{2}$ at $\mathrm{Ka}=8767$ (each panel shows approximately $20 l_{F} \times 30 l_{F}$ ); for $\mathrm{H}_{2}$ the normalisation is four times the laminar value (rather than ten). The reaction rate from the distributed supernova flame [Aspden et al., 2008a] is shown for comparison (the panel shows approximately $40 l_{F} \times 60 l_{F}$ ), which is normalised by one-fifth of the corresponding laminar value.

figure 3 reinforces this similarity by comparing the reaction rates from the distributed supernova flame with hydrogen fuel consumption rate and heat release (now normalised by four times the laminar values rather than ten). The peak reaction rates in the hydrogen case are lower than those of the moderate turbulent cases, but remain higher than in the one-dimensional laminar flame. This is in contrast to the supernova flame that showed a decrease in local fuel consumption rate; this can be explained as the suppression of Lewis number effects, and will be discussed further in section 4 .

\subsection{Turbulent flame speeds}

Turbulent flame speeds are shown both as a function of time and as a function of Ka in figure 4 (vertical lines in the latter denote the standard deviation after reaching a statistically-steady state). Note that at such extreme levels of turbulence, tens of integral length eddy turnover times are required for the flame to reach a statistically-steady state, and closer to 100 for the highest cases. The horizontal lines denote the mean and averaging period; in the following sections, all data has been temporally averaged in time using around 100 time points evenly distributed over the corresponding time periods. Power law scaling (as a function of Ka) can be derived in analogy with Damköhler [1940] for the distributed limit of turbulence-driven mixing, which is shown by the dotted lines (see also Peters [2000]). Predicting a turbulent flame speed $s_{T}=\sqrt{D_{T} / \tau_{T}}$, where $D_{T}=\alpha u^{\prime} l$, is a turbulent diffusion for some constant $\alpha$ and (turbulent) chemical time scale $\tau_{T}$ (both to be determined), three equivalent expressions can be derived

$$
\frac{s_{T}}{s_{F}} \propto \Lambda^{2 / 3} \mathrm{Ka}^{1 / 3}, \quad \frac{s_{T}}{s_{F}} \propto \Lambda \mathrm{Da}^{-1 / 2}, \quad \text { or } \quad \frac{s_{T}}{s_{F}} \propto \Lambda^{1 / 2} \Upsilon^{1 / 2},
$$

where the constant of proportionality in all three cases is $\alpha^{1 / 2} \mathrm{Da}_{T}^{-1 / 2}$; note the latter can be written as $\left(\operatorname{Re} / \operatorname{Re}_{F}\right)^{1 / 2}$ for $\operatorname{Re}_{F}=s_{F} l_{F} / \nu$. It is as yet unclear why both hydrogen and methane flames appear to follow this scaling law in a regime where it is not intended to apply (other than a simple dimensional necessity) followed by an apparent transition, after which the flame speeds may again follow the scaling law, especially since distributed burning behaviour is only observed for hydrogen at $\mathrm{Ka}=8767$. It should be be borne in mind that the present measure of turbulent flame speed is a global-consumption-based metric, and will depend on the size of the domain (which is usually not considered). Extending the domain without changing the integral length scale is unlikely to result in a simple periodic replica of the present flame; there is a greater volume into which the flame can develop, leading to greater flame surface area, and therefore greater turbulent flame speed by the present global-consumption-based metric. 

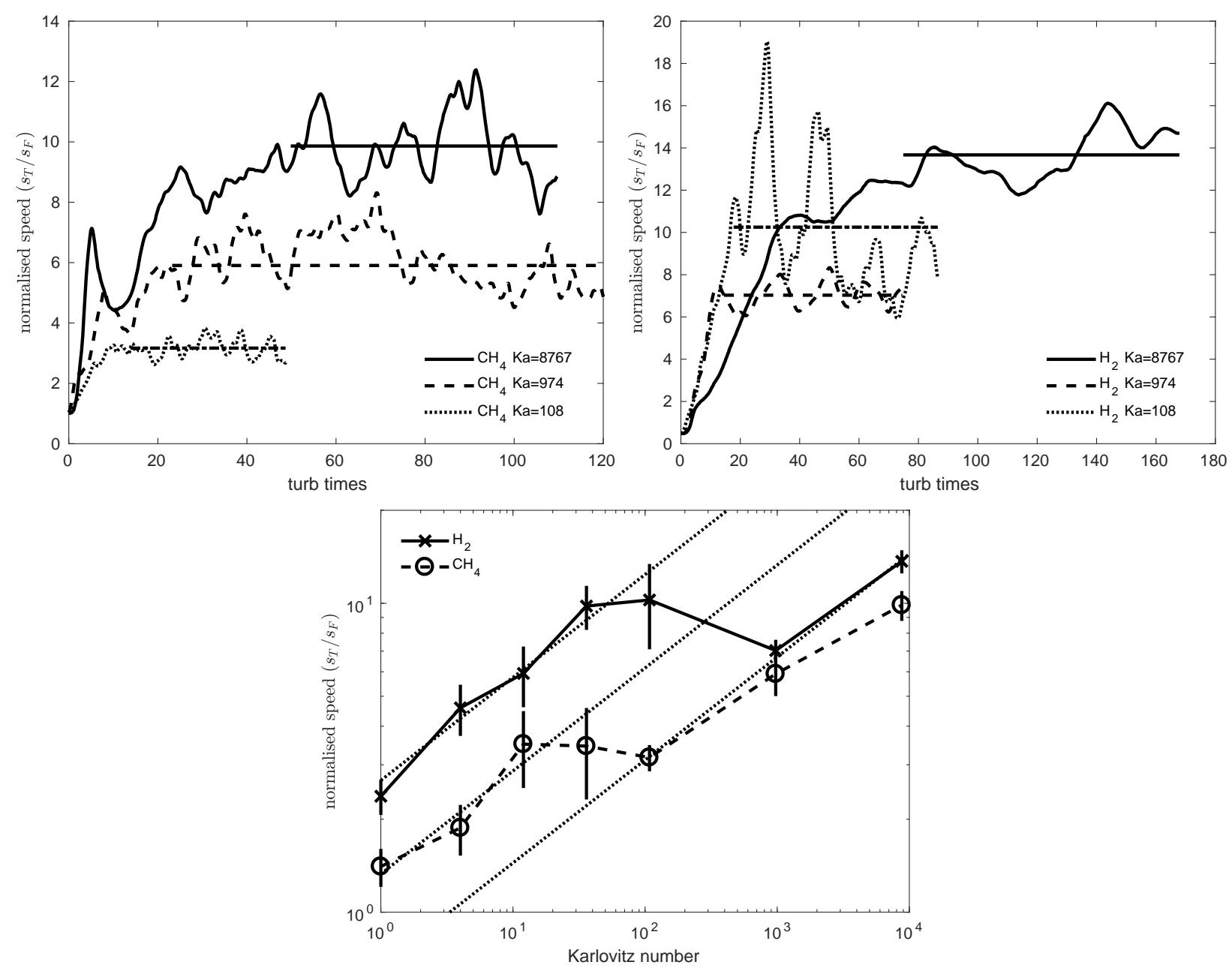

Figure 4: Top: normalised turbulent flame speeds $s_{T} / s_{F}$ as a function of turbulent eddy turnover times for methane (left) and hydrogen (right); horizontal lines denote the average and period treated as steady-state. Bottom: Normalised turbulent flame speed as a function of Ka; the vertical lines show one standard deviation about the mean, and the dotted lines show the scaling $s_{T} / s_{F} \propto \mathrm{Ka}^{1 / 3}$ (for fixed $\Lambda$ ), with three different constants of proportionality.

\subsection{Thickening factor}

A local thickening factor was previously defined [Aspden et al., 2016] analogously to thermal thickness as the ratio of the conditional means of temperature gradients as

$$
\Theta_{n}(T)=\frac{<\nabla T(\xi) \mid \xi=T>_{\mathrm{Ka}=1}}{<\nabla T(\xi) \mid \xi=T>_{\mathrm{Ka}=n}}
$$

where the normalisation by the conditional mean at $\mathrm{Ka}=1$ is used (in preference to the laminar profile) to account for the thermodiffusive instability in the hydrogen flames. In that paper, the methane flames were shown to be broadened in the preheat region, unlike the hydrogen flames, which became progressively thinner with increasing Ka due to generation of more highly-curved flame surface by turbulence thereby enhancing the thermodiffusive effects. The thickening factor in the present flames are compared in figure 5 with these previous lower Ka cases; the methane flames are broadened further in the preheat region with increasing $\mathrm{Ka}$, but seem to remain thin at $\mathrm{Ka}=8767$ in the post-flame region (the vertical dashed line indicates the location of peak HR). The thinning trend in hydrogen is reversed in the preheat region at higher Ka, but remain thinner in the post-flame region. This thinning in the post-flame region may be due in part to the normalisation, where the temperature profile $\mathrm{Ka}=1$ flame (as for the laminar flame) has a long tail at high temperatures (i.e. small gradients), resulting in a smaller $\Theta$ due a modified post-flame structure at high Ka. Furthermore, it appears that the thickening factor does not reflect the visual thickening observed in figure 2 ; as 

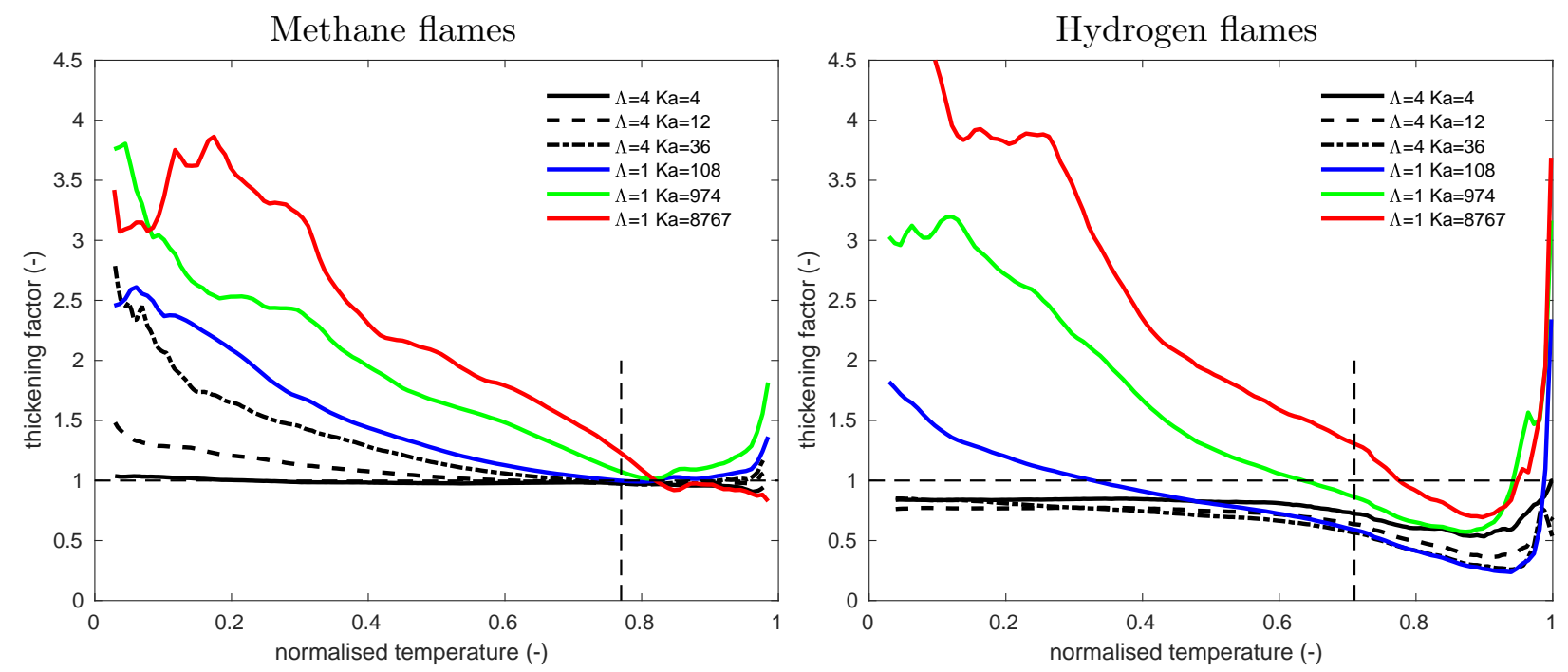

Figure 5: Thickening factor $\Theta(T)$ for $\mathrm{CH}_{4}$ (left) and $\mathrm{H}_{2}$ (right) compared with previous lower Ka cases from Aspden et al. [2015, 2016], which are shown in black.

the turbulence intensity increases, the flame becomes less flamelet-like, turbulent mixing maintains strong local gradients distributed over a broad region in space, which is compounded by conditioning on temperature without accounting for the broadening of the temperature field itself. At the highest $\mathrm{Ka}$, this metric becomes less reliable in the preheat region due to resolution limitations; the simulation with refined computational grid demonstrates that this sensitivity is predominantly observed in the preheat region, but is slight in the region of the flame (see figure 12 in the appendix).

\subsection{Reaction rates}

The response of reaction rates to turbulence is considered using conditional means of fuel consumption rate and heat release rate in figure 6. One-dimensional flame solutions are also presented for comparison: the red line denotes the laminar flame and the blue line denotes the unity Lewis number flame. The dashed magenta lines were obtained by one-dimensional calculations of the laminar flame where each diffusion coefficient has been supplemented by a constant turbulent diffusion (i.e. the diffusion coefficient $D_{i}$ for species $i$ was increased by $D_{T}$, where $D_{T}$ is a turbulent diffusion coefficient, with a corresponding thermal term to ensure unity Lewis number of the supplementary terms); $D_{T}$ was gradually increased in magnitude (giving the different dashed magenta lines) until the profile stopped changing, and that limiting case is shown by the solid magenta line.

At low-to-moderate Ka, the methane profile is close to the laminar profile, but at the highest Ka the profile appears to have shifted toward higher temperatures, with a slight increase in peak magnitude (consistent with figure 2). A more pronounced response is observed in the hydrogen flames; at low-tomoderate $\mathrm{Ka}$, there is substantial heat release rate in the preheat region (due to the thermodiffusive instability exaggerated by moderate turbulence), whereas at higher Ka the distribution is narrower, with a peak that again has shifted to higher temperatures, consistent with the peak temperature in the one-dimensional profiles, again indicative of suppression of Lewis number effects. Importantly, note the similarity between the hydrogen flames at $\mathrm{Ka}=974$ and 8767 ; this suggests that the conditional mean is tending to a limiting distribution, which we argue is to be anticipated as a characteristic of distributed burning.

\subsection{Species mass fraction distributions}

For the methane flames, the turbulent response of conditional means of species mass fractions remain consistent with the classification set out in Aspden et al. [2016]; figure 7(left column) presents conditional means of mass fractions for $\mathrm{CH}_{4}, \mathrm{H}_{2}$ and $\mathrm{H}_{2} \mathrm{O}_{2}$ at $\mathrm{Ka}=8767$ (conditional means for all species and $\mathrm{Ka}$ are provided as supplementary material). The fuel distribution is close to linear for all profiles, 

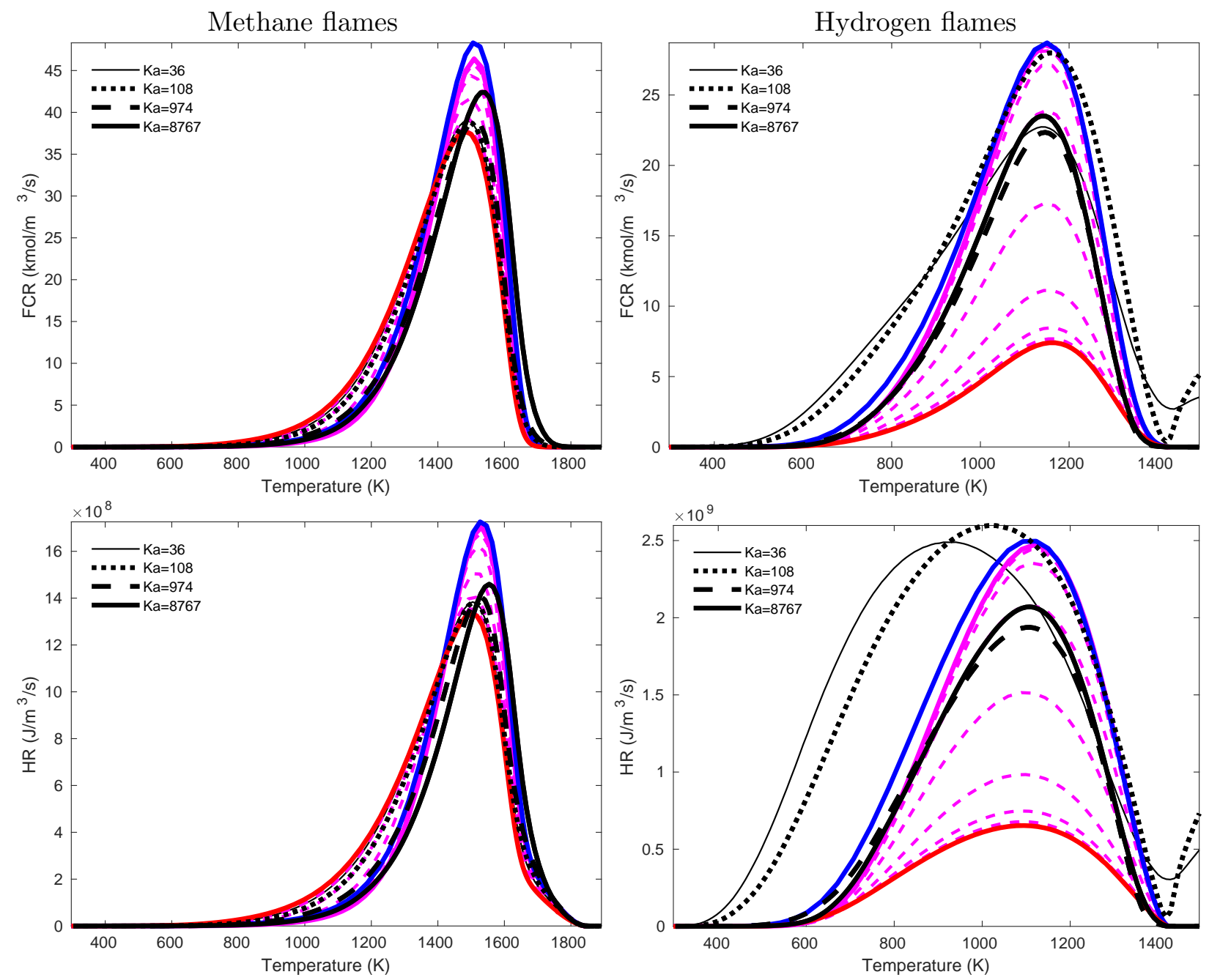

Figure 6: Conditional means of fuel consumption rate and heat release for $\mathrm{CH}_{4}$ (left) and $\mathrm{H}_{2}$ (right) flames. Black lines are the conditional means from the simulations, red is the one-dimensional laminar flame profile, blue is the one-dimensional unity Lewis number profile, and the dashed magenta lines are one-dimensional laminar flames with an added turbulent diffusion of increasing magnitude with the limiting case shown as a solid line.

but there is the suggestion that the turbulent profile is slightly higher than the one-dimensional profiles (not seen in the lower Ka cases). The other two species present a strong response in the preheat region; specifically, turbulence dominates species diffusion at high Ka. The standard deviation decreases with $\mathrm{Ka}$, and the distribution not only transitions from that comparable with the laminar profile (red) towards the unity Lewis number profile (blue), but also appears to reach a state that is distinct from all three of the one-dimensional profiles. Aspden et al. [2016] suggested that a temperature-dependent diffusion coefficient may account for the difference, and that it may be due the effect of dilatation on turbulence through the flame; there may also be a change in chemical time scale. Other species with distributions notably distinct are $\mathrm{C}_{2} \mathrm{H}_{2}, \mathrm{HCCOH}$ and $\mathrm{CO}$ (see supplementary material).

A similar response is found for intermediate species in the hydrogen flames, but there is a significantly different response in the fuel distribution; figure 7(right column) presents conditional means of species mass fraction for $\mathrm{H}_{2}, \mathrm{H}$ and $\mathrm{H}_{2} \mathrm{O}_{2}$ at $\mathrm{Ka}=8767$. The fuel distribution has moved away from the (low Le) one-dimensional laminar flame (that lies below the diagonal) towards the unity Lewis number profile that aligns with the diagonal mixing line. The response of $\mathrm{H}$ is characteristic of $\mathrm{O}$ and $\mathrm{OH}$ (again, all species are presented as supplementary material), where the enhanced radical pool at low-to-moderate temperatures observed at lower Ka [see Aspden et al., 2015, and supplementary material] is suppressed at $\mathrm{Ka}=8767$. A strong response of $\mathrm{H}_{2} \mathrm{O}_{2}$ is observed in the preheat region, and again appears to tend to a limit distinct from the three one-dimensional profiles. The standard 
Methane flames
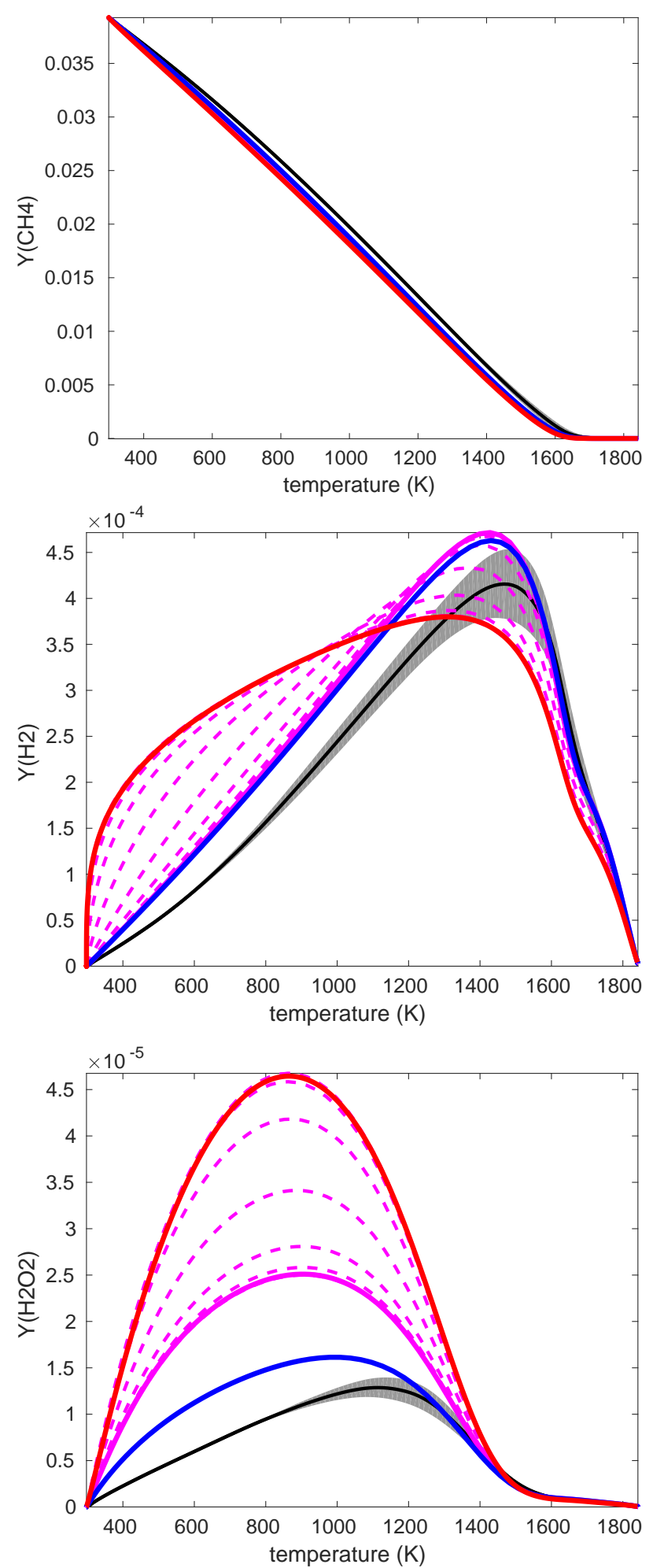

Hydrogen flames
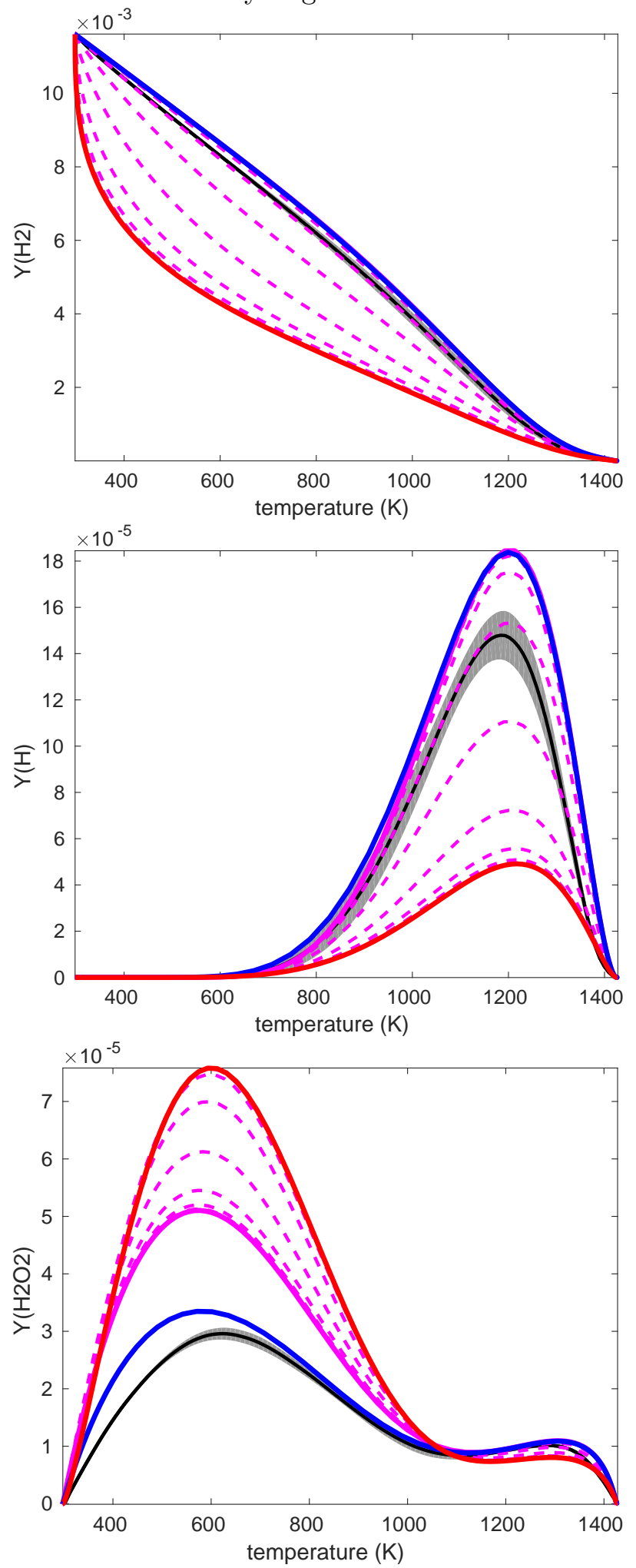

Figure 7: Conditional means of species mass fraction for $\mathrm{CH}_{4}$ (left) and $\mathrm{H}_{2}$ (right) flames at $\mathrm{Ka}=8767$; see figure 6 for a description of the line styles, with the addition of one standard deviation about the mean shown in grey. 


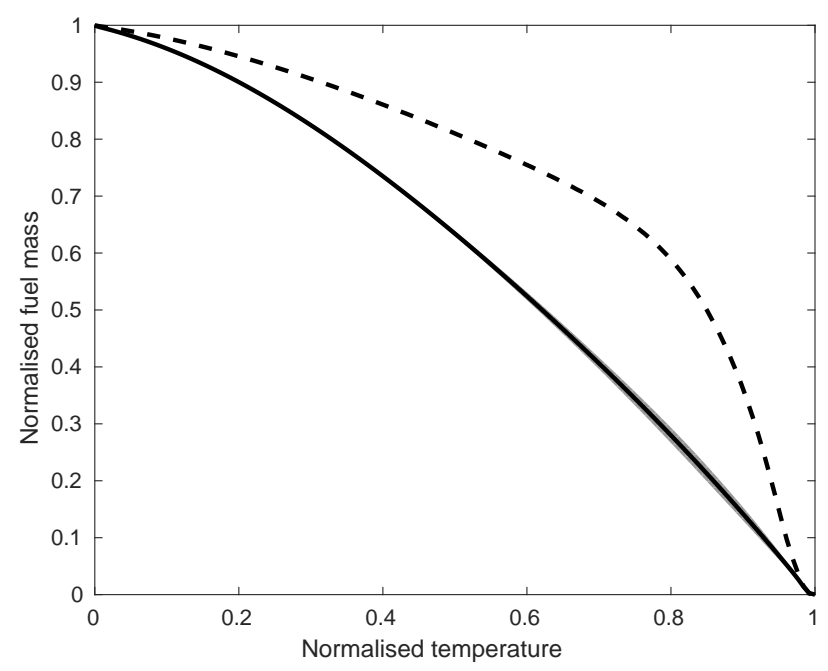

Figure 8: Conditional mean of normalised fuel mass from the distributed supernova flame at Ka $=266$ [Aspden et al., 2008a]; the conditional mean is the solid line with one standard deviation in grey, and the laminar flame profile is shown by the dashed line.

deviation is also significantly reduced, almost to zero.

\section{Discussion and Conclusions}

Numerical simulations at extreme levels of turbulence have shown a transition to distributed burning in lean premixed hydrogen flames. The phenomenology of the transition is similar to that reported in an astrophysical context [Aspden et al., 2008a]; there are no indications that suggest other fuels should not undergo a similar transition at sufficiently intense turbulence levels. Unrealistically-high turbulence conditions were required to observe the transition, which is argued to be primarily due to dilatation and the higher density ratio between unburned and burned conditions (the density ratio is less than two for the supernova case, about four for hydrogen, and over six for methane); this effect was not observed in constant-density reaction wave propagation by $\mathrm{Yu}$ and Lipatnikov [2017], for example. The effect of density ratio may also be compounded by the thickness of the reaction zone relative to the thermal thickness; the reaction thickness in the methane flame is relatively narrower than in the hydrogen flame, so turbulence has to survive deeper into the methane flame to disrupt the reaction zone.

The key behaviour characteristic of distributed burning not previously observed in chemical flames is broadening of the reaction zone by turbulence; here, the heat release region was visually found to be broadened by around an order of magnitude. Thermodiffusive effects (in particular, the super-adiabatic regions and the decorrelation of FCR and HR) were found to be suppressed, if not eliminated, with the peak reaction rates falling from about fifteen times the peak laminar value to about four times. Distributed reaction rates that are higher than the corresponding laminar flame is different from the response found in the supernova flame, where the peak reaction rates were found to be much smaller. This can be attributed to global Lewis number by considering the fuel-temperature distribution, as discussed in Aspden et al. [2011b]. Hydrogen has a low Lewis number, and so the fuel-temperature distribution of the laminar flame lies below the linear mixing distribution. Strong turbulent mixing gives an effective unity Lewis number for all species (species and enthalpy are advected together in packets) so the distributed flame profile is close to linear - fuel concentrations are higher at the same temperature, so reaction rates and heat release increase. Conversely, for the supernova, the Lewis number is large, so the laminar flame profile lies above the linear mixing distribution (see figure 8), and turbulent mixing results in fuel concentrations that are lower at the same temperature, giving lower reaction rates.

Turbulent flame speeds were found to follow scaling laws at low-to-moderate Karlovitz numbers (despite not satisfying the assumptions made), followed by an apparent transition. Even at the 
extreme turbulence levels considered, there was insufficient evidence to draw solid conclusions about behaviour in the distributed burning regime, and will require significant further work to demonstrate the behaviour observed in the supernova flames [Aspden et al., 2010].

The species distribution tends towards a limit that is distinct from the one-dimensional profiles (laminar, unity Le, and turbulent diffusive limit), with a standard deviation of almost zero; Aspden et al. [2016] suggested that a temperature-dependent diffusion coefficient is required to account for dilatation through the flame. A change in distribution was observed in particular for species that experience low-temperature activity (e.g. $\mathrm{H}_{2} \mathrm{O}_{2}$ ), which is argued to result from molecular diffusion of mobile species such as $\mathrm{H}_{2}$ and/or $\mathrm{H}$ being overcome by turbulent mixing.

\subsection{Transition to distributed burning}

We continue to interpret the distributed burning regime as the limiting behaviour where turbulent mixing dominates species and thermal diffusion to drive flame propagation, as discussed in Aspden et al. [2011a], along with the consequences described therein. It is well-established that turbulence increases the flame surface area and exaggerates the thermodiffusive instability, but once turbulence is sufficiently intense, it can overcome dilatation effects to disrupt the reaction zone. We posit that the turbulently-mixed flame structure (i.e. chemical distribution and reaction rates conditioned on some measure of progress variable such as temperature) eventually reaches a limiting state (distinct from other one-dimensional distributions considered here) that is invariant under further increases in turbulent intensity. The turbulent flame speed and thickness will respond to such increases in turbulent intensity (and so spatial gradients will eventually become small) but the underlying flame structure will not change (in temperature space).

We argue that only turbulence-flame interactions around the flame scales, i.e. length scales comparable with the thermal and reaction thicknesses, are relevant to the transition; larger scales serve to move the turbulent flame structure around, and smaller scales are not dynamically important. Therefore, the relevant dimensionless quantity necessary for distributed burning is sufficiently high Karlovitz number, defined according to equation (1), because it appropriately characterises the turbulence in terms of the inertial subrange. As Karlovitz number increases, turbulence is able to penetrate further into the flame, until eventually it is sufficiently strong enough to overcome dilatation effects and mix the reaction zone quicker than it can burn. While these simulations have a particularly small integral length scale, they are representative of how turbulence at the same Karlovitz number would interact with the flame; the response to increasing the integral length scales at a fixed Karlovitz number in the distributed regime was demonstrated in Aspden et al. [2010], consistent with Zimont [1979].

The critical Karlovitz number necessary is fuel-dependent, and will be higher where the density jump across the flame is large (due to suppression of turbulence by dilatation); it is not universal, and it is certainly not 100 . The global Lewis number (that of the deficient species) will also affect this critical Karlovitz number. The transition to distributed burning increases the local reaction rates for low Le (and vice versa for high Le), which suggests that the critical Karlovitz number required to transition will be lower at high Le; if the reaction rates decrease, then there is more time for turbulence to interact with the reaction zone, allowing it to mix before it can burn. Moreover, the Karlovitz number defined in equation (1) is based on the laminar flame properties (or indeed freely-propagating values in the case of hydrogen), but in a distributed flame, the global Lewis number becomes irrelevant because molecular diffusion is dominated by turbulent mixing; if one were to artificially alter the global Lewis number, the reference flame properties would be different, but the corresponding distributed flames should be anticipated to be identical for the same turbulence conditions (not the same Ka). This suggests that the critical Karlovitz number could be evaluated based on a one-dimensional calculation that somehow accounts for the subtle interaction of an appropriate turbulent mixing model (with an effectively unity Lewis number) and the resulting chemical time scale. Furthermore, the use of the freely-propagating reference values may only be relevant for the thin reaction zone.

We contend that the present hydrogen flame at the highest Ka has indeed achieved the distributed burning regime. Note that the limiting case is not confirmed definitively by the present simulations as that would require yet higher Ka to establish invariant behaviour (although the $\mathrm{Ka}=974$ case is not substantially different). What is confirmed by the present simulations is a change in flame structure, 

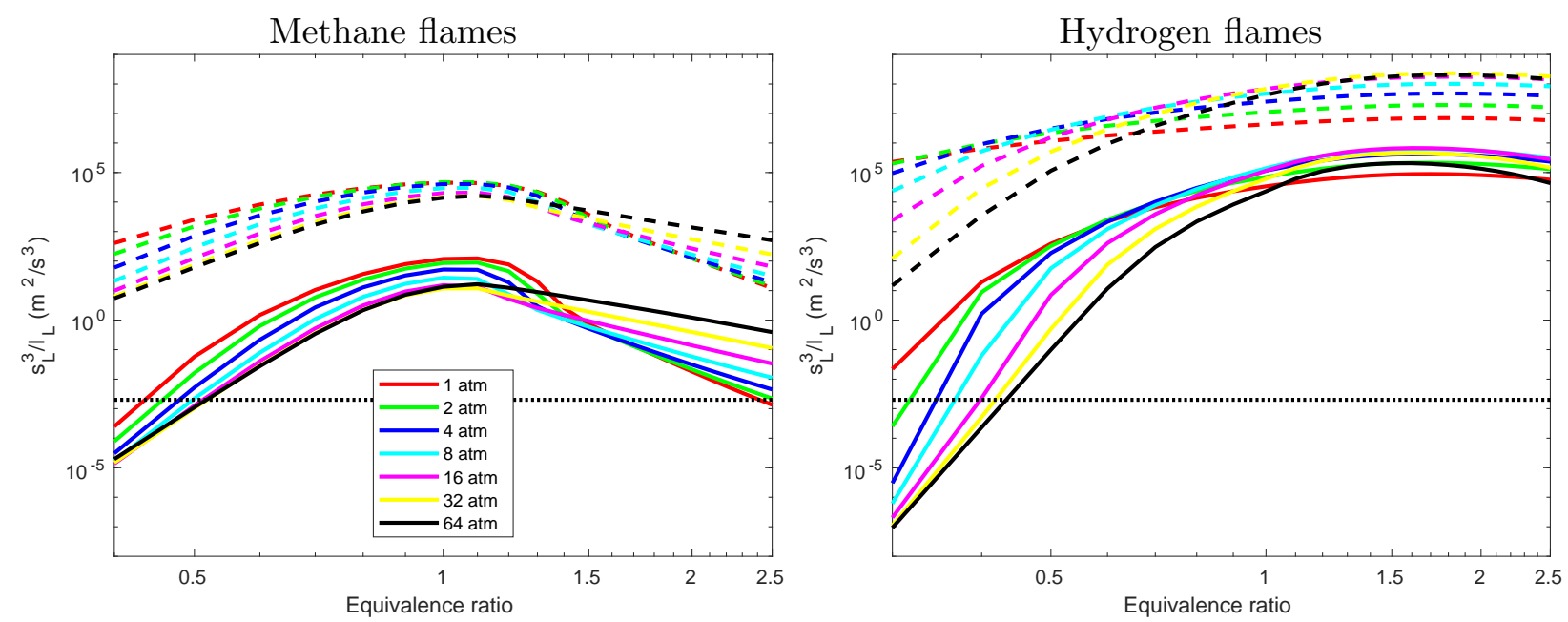

Figure 9: Laminar flame properties from methane (left) and hydrogen (right) flames; solid and dashed lines denote reactant temperatures of $300 \mathrm{~K}$ and $800 \mathrm{~K}$, respectively. Maximising Karlovitz number is equivalent to minimising $s_{L}^{3} / l_{L}$; given sample conditions of $u^{\prime}=10 \mathrm{~m} / \mathrm{s}$ at $l=5 \mathrm{~mm}$, a Karlovitz number in excess of 10,000 can be achieved for reactant conditions with $s_{L}^{3} / l_{L}$ below $0.002 \mathrm{~m}^{2} / \mathrm{s}^{3}$, as shown by the dotted horizontal line.

especially a spatial broadening of the reaction zone by turbulence, accompanied by a (Le-dependent) change in magnitude, and a distinct chemical distribution.

\subsection{Potential for realising distributed burning}

Whether or not there is a practical use for distributed flames remains to be seen, but it remains a phenomenon of academic interest, in particular as the limiting case of high-Karlovitz-number turbulenceflame interaction. The conditions required here for distributed burning are unphysical and unrealisable in practice, but there are steps that could be taken that may lead to distributed burning. To explore potential conditions, a series of one-dimensional laminar flame calculations were run using Cantera [Goodwin et al., 2014] to obtain the laminar flame speed $\left(s_{L}\right)$, the thermal thickness $\left(l_{L}\right)$ and the density ratio $\left(P=\rho_{u} / \rho_{b}\right)$ between reactant and products. Reactant temperatures were varied between $300 \mathrm{~K}$ and $800 \mathrm{~K}$, pressures from $1 \mathrm{~atm}$ to $64 \mathrm{~atm}$, and equivalence ratios from 0.3 for $\mathrm{H}_{2}$ and 0.4 for $\mathrm{CH}_{4}$ (and $\mathrm{C}_{12} \mathrm{H}_{26}$; see supplementary material) up to 2.5 .

Since the strength of the turbulence that interacts at the flame scale depends solely on Ka, then for given turbulence conditions $u^{\prime}$ and $l$, finding reactant conditions favourable for distributed burning (i.e. maximising Ka) is equivalent to minimising $s_{L}^{3} / l_{L}$. Starting with example realisable conditions, say $u^{\prime}=10 \mathrm{~m} / \mathrm{s}$ at $l=5 \mathrm{~mm}$, then obtaining a Karlovitz number of 10,000 requires the ratio $s_{L}^{3} / l_{L}$ to be below $0.002 \mathrm{~m}^{2} / \mathrm{s}^{3}$. This ratio is shown for methane and hydrogen flames as a function of equivalence ratio at $T=300 \mathrm{~K}$ (solid lines) and $800 \mathrm{~K}$ (dashed lines) for all pressures (all other data is presented as supplementary material). The figure shows that the likelihood of realising distributed burning can be increased by increasing pressure, decreasing equivalence ratio, and decreasing reactant temperature. Preheating reduces the density ratio, and therefore the impact of dilatation on turbulence through the flame, but increasing the reactant temperature from $300 \mathrm{~K}$ to $800 \mathrm{~K}$ only reduces the density ratio by a factor of about two. The increase in flame speed is closer to two orders of magnitude, and so is likely to far outweigh the benefit of reduced density ratio; preheating is unlikely to be favourable for the transition to distributed burning.

MSD and JBB were supported by the DOE Applied Mathematics Research Program of the DOE Office of Advanced Scientific Computing Research under the U.S. Department of Energy Contract No. DE-AC02-05CH11231, which included computational resources at the National Energy Research Scientific Computing Center (NERSC). 

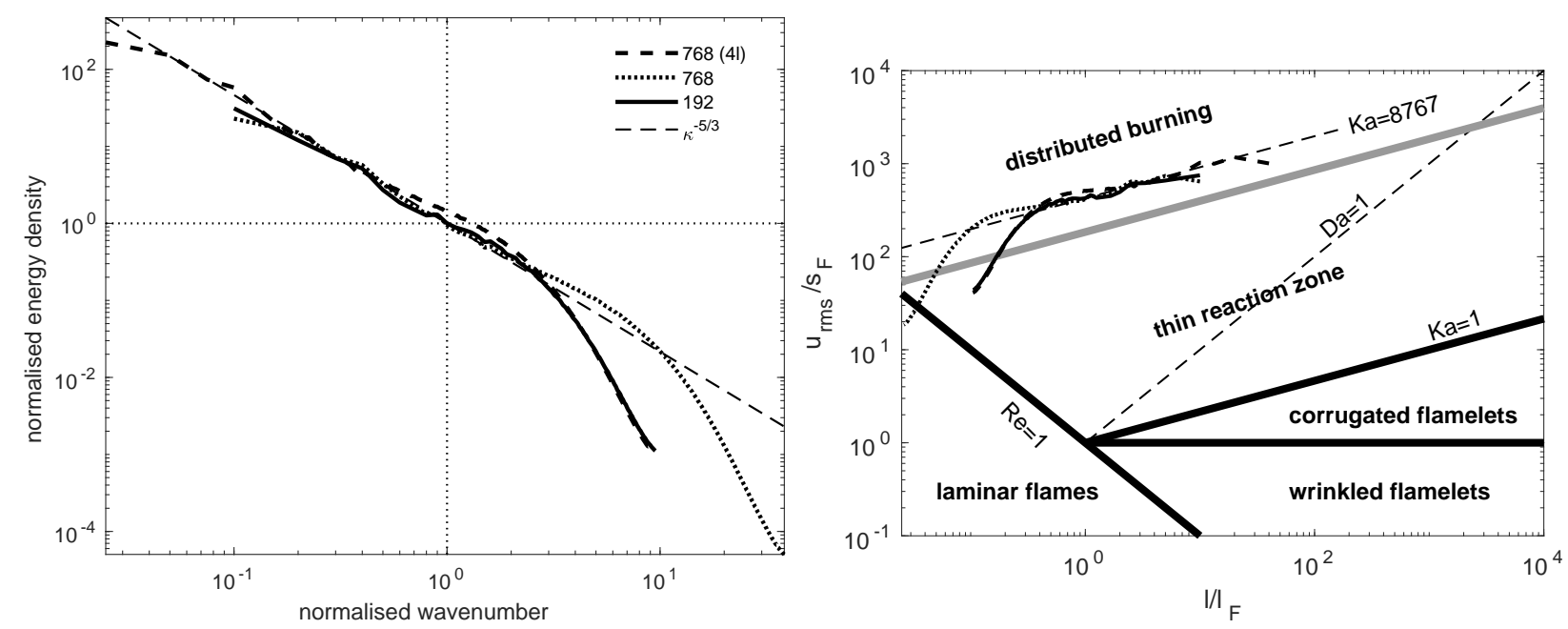

Figure 10: Normalised kinetic energy density spectrum as a function of wavenumber normalised by the thermal thickness of the laminar flame (left), and the same recast schematically on the regime diagram (right).

\section{Appendix A}

To explore the potential consequences of the lack of resolution on the turbulence, this appendix presents simulations of homogeneous isotropic turbulence in a triply-periodic cube at the same (reactant) conditions as the hydrogen flame case at the highest Karlovitz number. One simulation was run at $192^{3}$, corresponding directly to the same resolution as the flame simulation, another at $768^{3}$ with the same integral length scale and therefore four times the resolution, and a final case at $768^{3}$ with an integral length scale four times greater (denoted $4 l$ ) and therefore matching the cell size; in this last case, the magnitude of the forcing was increased to match the energy dissipation rate, which corresponds to matching the Karlovitz number.

The kinetic energy density spectra for the three cases are shown in figure 10 (left), where the wavenumber has been normalised by the laminar flame thickness and the energy density has been normalised by that at the flame scale in the $192^{3}$ case. Note the coincidence of the inertial subranges, however short, and that they closely follow the expected five-thirds cascade. The inertial subrange of the $192^{3}$ case is clearly truncated by the lack of small-scale resolution, however this occurs at wavenumbers greater than the flame scale, which supports the argument that the scales represented on the grid are adequate to capture turbulence-flame interactions. The energy density spectra have also been recast schematically on the regime diagram by noting $\sqrt{\kappa E}$ is representative of the velocity scale, where $\kappa$ and $E$ are wavenumber and energy density, respectively. This illustrates how the inertial subrange follows a constant Karlovitz line (shown by the dashed black line; referred to as turbulence lines by Poinsot and Veynante [2005], for example) because the energy dissipation rate is constant throughout $\left(\mathrm{Ka} \sim \varepsilon^{1 / 2} \sim\right.$ const), and where the lack of resolution compromises the calculation (the inertial range should continue to follow the constant Karlovitz line), again lending support to the argument that the lack of resolution will not be detrimental to resolving the flame physics. It is this coincidence of the inertial subrange with the constant Karlovitz line that supports the use of socalled small-eddy simulation approach for turbulent-flame interactions, and for the use of the present definition of $\mathrm{Ka}$ (rather than one involving Kolmogorov scales or viscosity); specifically, it is the appropriate definition characterising turbulence-flame interactions, not one based on the Kolmogorov time scale and the assumption that $\nu \approx s_{F} l_{F}$. The simulations presented here are underresolved, which leads to a smaller effective Reynolds number but the Karlovitz number (appropriately defined) is the same. 


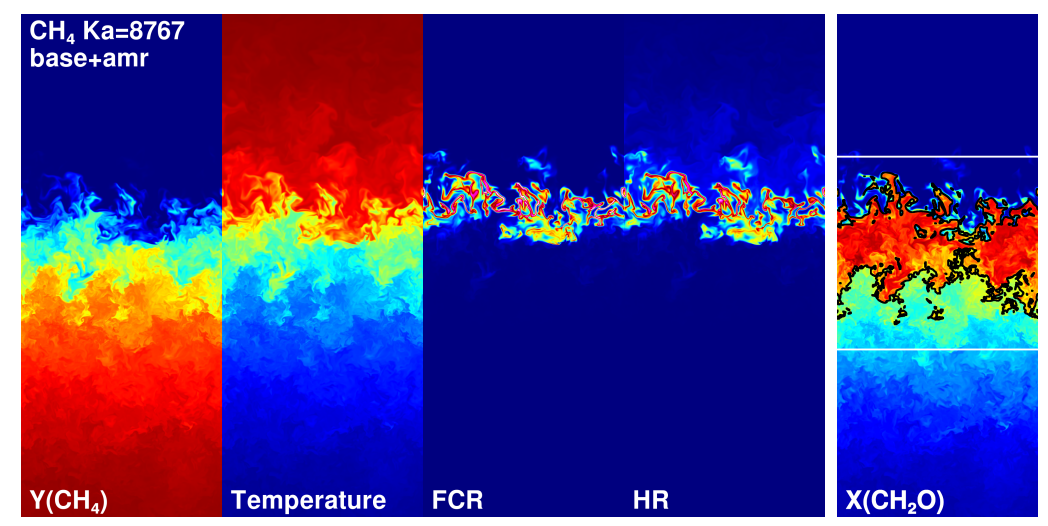

Figure 11: Slices of fuel mass fraction, temperature, fuel consumption rate, and heat release for the $\mathrm{CH}_{4}$ flame at $\mathrm{Ka}=8767$ run with adaptive mesh refinement (for comparison with figure 2), along with formaldehyde mole fraction, which was used for gridding criterion (the black contour plus a 16cell buffer resulted in the region between the two horizontal white lines being refined, capturing the reaction layer and a substantial region ahead of it).
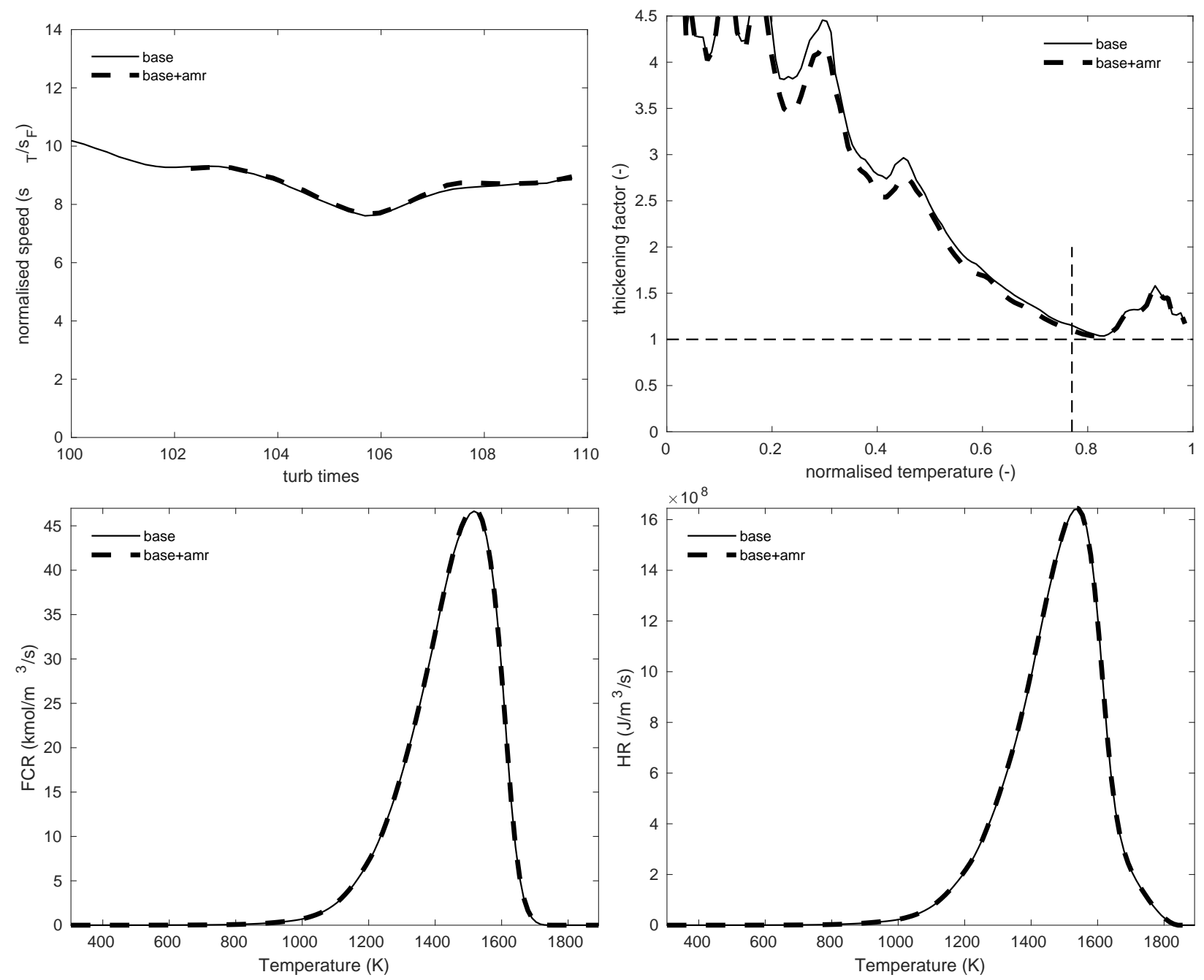

Figure 12: Comparison of turbulent flame speeds, thickening factor, and conditional means of fuel consumption rate and heat release with adaptive mesh refinement for the $\mathrm{CH}_{4}$ flame at $\mathrm{Ka}=8767$. 


\section{Appendix B}

To establish the consequences for not completely resolving the spatial scales down to the Kolmogorov length scale in the methane flame at the highest Karlovitz number, this appendix presents an additional simulation with higher resolution; this was achieved by restarting the calculation with one level of adaptive mesh refinement around the flame. Refinement was added based on formaldehyde mole fraction (above $5 \times 10^{-4}$ ) with an additional buffer of 16 computational cells, following Aspden et al. [2011a] to allow the higher wavenumbers to become populated; at this higher resolution there are over 38 computational cells across the thermal thickness of the laminar flame.

Figure 11 shows slices of fuel, temperature, fuel consumption rate and heat release for the case with adaptive mesh refinement (for comparison with figure 2), along with formaldehyde mole fraction with the gridding threshold contour shown in black and the resulting region of increase resolution shown by the two white lines. While it is unreasonable to expect that the turbulence structure is exactly the same, the general flame features appear unaffected by the increase in resolution.

Figure 12 shows normalised turbulent flame speed, thickening factor and conditional means of fuel consumption rate and heat release (conditioned on temperature); in each case, the base grid is shown as a thin solid line, and the case with adaptive mesh refinement is shown as a thick dashed line. The temporal averaging was performed over the final two-thirds of the restarted run (corresponding to about five eddy turnover times), and the same time period was also used at the lower resolution. The turbulent flame speed (a global metric) agrees almost exactly between resolutions, and the conditional means of fuel consumption rate and heat release (local metrics) are indistinguishable. There do appear to be differences in the thickening factor between the two resolutions, specifically, the thickening factor is lower at the higher resolution, indicative of slightly higher gradients; both the higher gradient and disparity are not particularly surprising, especially for a higher moment in a short period of temporal averaging. Ultimately, this higher resolution simulation demonstrates that the effect of the apparent lack of resolution is small.

\section{References}

A. J. Aspden. A numerical study of diffusive effects in turbulent lean premixed hydrogen flames. $P$. Combust. Inst., 36:1997 - 2004, 2017. ISSN 1540-7489. doi: https://doi.org/10.1016/j.proci.2016. 07.053 .

A. J. Aspden, J. B. Bell, M. S. Day, S. E. Woosley, and M. Zingale. Turbulence-flame interactions in type Ia supernovae. Astrophys. J., 689:1173-1185, December 2008a.

A. J. Aspden, N. Nikiforakis, S. B. Dalziel, and J. B. Bell. Analysis of implicit LES methods. Comm. App. Math. Comput. Sci., 3(1):101, December 2008b.

A. J. Aspden, J. B. Bell, and S. E. Woosley. Distributed flames in type Ia supernovae. Astrophys. J., 710:1654-1663, 2010.

A. J. Aspden, M. S. Day, and J. B. Bell. Turbulence-flame interactions in lean premixed hydrogen: transition to the distributed burning regime. J. Fluid Mech., 680:287-320, 2011a.

A. J. Aspden, M. S. Day, and J. B. Bell. Lewis number effects in distributed flames. P. Combust. Inst., 33(1):1473 - 1480, 2011b.

A. J. Aspden, M. S. Day, and J. B. Bell. Turbulence-chemistry interaction in lean premixed hydrogen combustion. P. Combust. Inst., 35(2):1321 - 1329, 2015. ISSN 1540-7489.

A. J. Aspden, M. S. Day, and J. B. Bell. Three-dimensional direct numerical simulation of turbulent lean premixed methane combustion with detailed kinetics. Combust. Flame, 166:266 - 283, 2016.

A. J. Aspden, J.B. Bell, M.S. Day, and F.N. Egolfopoulos. Turbulence-flame interactions in lean premixed dodecane flames. P. Combust. Inst., 36:2005 - 2016, 2017. ISSN 1540-7489. 
M. Baum, T. J. Poinsot, D. C. Haworth, and N. Darabiha. Direct numerical simulation of $\mathrm{H}_{2} / \mathrm{O}_{2} / \mathrm{N}_{2}$ flames with complex chemistry in two-dimensional turbulent flows. J. Fluid Mech., 281:1-32, 1994.

J. H. Chen and H. Im. Stretch effects on the burning velocity of turbulent premixed hydrogen/air flames. Proc. Combust. Inst., 28(1):211-218, 2000.

G. Damköhler. Der Einfluss der Turbulenz auf die Flammengeschwindigenkeit in Gasgemischen. $Z$. Elektrochem, 46:601 - 652, 1940.

M. S. Day and J. B. Bell. Numerical simulation of laminar reacting flows with complex chemistry. Combust. Theor. Model., 4:535-556, 2000.

M. J. Dunn, A. R. Masri, R. W. Bilger, and R. S. Barlow. Finite rate chemistry effects in highly sheared turbulent premixed flames. Flow Turbul. Combust., 85(3-4):621-648, 2010. ISSN 1386-6184. doi: $10.1007 / \mathrm{s} 10494-010-9280-5$.

T. Echekki and J. H. Chen. Unsteady strain rate and curvature effects in turbulent premixed methaneair flames. Combust. Flame, 106(1-2):184-202, 1996.

A. Ern and V. Giovangigli. Optimized transport algorithms for flame codes. Combust. Sci. and Tech., 118:387-396, 1996. See also http://www.cmap.polytechnique.fr/www.eglib/.

M. Frenklach, H. Wang, M. Goldenberg, G. P. Smith, D. M. Golden, C. T. Bowman, R. K. Hanson, W. C. Gardiner, and V. Lissianski. GRI-Mech - an optimized detailed chemical reaction mechanism for methane combustion. Technical Report GRI-95/0058, Gas Research Institute, 1995. http: //www.me.berkeley.edu/gri_mech/.

D. G. Goodwin, H. K. Moffat, and R. L. Speth. Cantera: An object-oriented software toolkit for chemical kinetics, thermodynamics, and transport processes. http://www.cantera.org, 2014.

F. F. Grinstein, L. G. Margolin, and W. J. Rider. Implicit Large Eddy Simulation. Cambridge University Press, 2007.

S. Lapointe, B. Savard, and G. Blanquart. Differential diffusion effects, distributed burning, and local extinctions in high Karlovitz premixed flames. Combust. Flame, 162(9):3341-3355, 2015.

J. Li, Z. Zhao, A. Kazakov, and F. L. Dryer. An updated comprehensive kinetic model of hydrogen combustion. Int. J. Chem. Kinet., 36(10):566-575, 2004.

T. Nilsson, H. Carlsson, R. Yu, and X.-S. Bai. Structures of turbulent premixed flames in the high Karlovitz number regime - DNS analysis. Fuel, 216:627 - 638, 2018.

A. Nonaka, J. B. Bell, M. S. Day, C. Gilet, A. S Almgren, and M. L. Minion. A deferred correction strategy for low mach number flow with complex chemistry. Combust. Theor. Model., 16(6):10531088, 2012.

N. Peters. Turbulent Combustion. Cambridge University Press, 2000.

T. Poinsot and D. Veynante. Theoretical and Numerical Combustion, 2nd edition. Edwards, 2005.

A. Y. Poludnenko and E. S. Oran. The interaction of high-speed turbulence with flames: Global properties and internal flame structure. Combust. Flame, 157(5):995-1011, 2010.

B. Savard and G. Blanquart. Broken reaction zone and differential diffusion effects in high Karlovitz n- $\mathrm{C}_{7} \mathrm{H}_{16}$ premixed turbulent flames. Combust. Flame, 162(5):2020-2033, 2015.

A. W. Skiba, T. M Wabel, C. D. Carter, S. D. Hammack, J. E. Temme, and J. F. Driscoll. Premixed flames subjected to extreme levels of turbulence part I: Flame structure and a new measured regime diagram. Combust. Flame, 189:407-432, 2018. 
C. A. Z. Towery, A. Y. Poludnenko, J. Urzay, J. O'Brien, M. Ihme, and P. E. Hamlington. Spectral kinetic energy transfer in turbulent premixed reacting flows. Phys. Rev. E, 93(5):053115, 2016.

A. Trouvé and T. Poinsot. The evolution equation for the flame surface density in turbulent premixed combustion. J. Fluid Mech., 278:1-31, 1994.

H. Wang, E. R. Hawkes, B. Savard, and J. H. Chen. Direct numerical simulation of a high $\mathrm{Ka} \mathrm{CH}_{4} /$ air stratified premixed jet flame. Combust. Flame, 193:229-245, 2018.

R. Yu and A. N. Lipatnikov. Direct numerical simulation study of statistically stationary propagation of a reaction wave in homogeneous turbulence. Phys. Rev. E, 95(6):063101, 2017.

B. Zhou, C. Brackmann, Z. Wang, Z. Li, M. Richter, M. Aldén, and X.-S. Bai. Thin reaction zone and distributed reaction zone regimes in turbulent premixed methane/air flames: Scalar distributions and correlations. Combust. Flame, 175:220-236, 2017.

V. L. Zimont. Theory of turbulent combustion of a homogeneous fuel mixture at high Reynolds numbers. Combust. Explos. Shock Waves, 15(3):305-311, 1979. 